\title{
Effects of early home-based strength and sensory- motor training after total hip arthroplasty: study protocol for a randomized controlled trial
}

Pika Krištof Mirt ( $\sim$ pikamirt@gmail.com)

General Hospital Novo Mesto: Splosna Bolnisnica Novo Mesto https://orcid.org/0000-0002-7051-2955

Vojko Strojnik

Univerza v Ljubljani Fakulteta za sport

Gregor Kavčič

General Hospital Novo Mesto: Splosna Bolnisnica Novo Mesto

Rihard Trebše

Valdoltra Orthopaedic Hospital: Ortopedska Bolnica Valdoltra

\section{Research Article}

Keywords: Total hip arthroplasty, Postoperative rehabilitation, Early postoperative exercise, Home-based training

Posted Date: November 1st, 2021

DOI: https://doi.org/10.21203/rs.3.rs-572172/v1

License: (c) (i) This work is licensed under a Creative Commons Attribution 4.0 International License. Read Full License 


\section{Abstract}

\section{Background}

Total hip arthroplasty (THA) is very effective in alleviating pain, but functional deficits persist up to a year following surgery. Regardless of standard physiotherapy programs, significant additional muscular atrophy and weakness occurs. Deficits in strength have serious adverse consequences for these patients with respect to physical function, the maintenance of independence and the requirement for revision surgery. Progressive resistance training in rehabilitation following THA has been shown to significantly enhance muscle strength and function. The fundamental principle is to progressively overload the exercised muscle as it becomes stronger. Different strength training protocols have been used at different times in the postoperative phase, in group or individual practices, with major differences being in centerbased and home-based programs with or without supervision. The primary objectives of our study are to evaluate whether an early postoperative home-based strength training protocol is feasible for all elective THA patients, does not cause major adverse effects and can improve patient functional outcomes at 3 months and 1 year following surgery.

\section{Methods/Design}

This study is a prospective multicenter randomized clinical trial to be conducted in the orthopedic departments of two Slovenian hospitals. In each hospital, 124 patients aged 60 or older with unilateral osteoarthritis, an ASA score between 1 and 3, signed informed consent form, and no terminal illness disabling rehabilitation participation will be randomly assigned to the intervention or control group. THA with an anterior approach will be performed. All patients will receive current standard physiotherapy during hospitalization. Patients in the intervention group will also learn strength and sensory-motor training exercises. Upon discharge all will receive USB drives with exercise videos, written exercise instructions and a training diary. Physiotherapists will perform the assessments (physical tests and the maximal voluntary isometric contraction assessment), and patients will fill out outcome assessment questionnaires (the Harris Hip Score and 36-Item Short Form Health Survey) at baseline and 1, 3 and 12 months after surgery.

\section{Discussion}

The main purpose of our study is to design a new standardized rehabilitation protocol with videos that will be effective, safe and accessible to all Slovenian THA patients.

\section{Trial registration}

ClinicalTrials.gov ID: NCT04061993. Protocol ID: PRT_PhD. Record Verification April 2021. https://clinicaltrials.gov/ct2/show/NCT04061993

\section{Background}


Total hip arthroplasty (THA) is one of the most widely performed and clinically successful surgical procedures, and the number of THA operations is rapidly increasing $1,2,3,4$. The number of THA procedures is expected to continue rising worldwide due to the wide range of indications for the procedure, and the prevalence of osteoarthritis $(\mathrm{OA})$ is expected to increase as the population ages; accordingly, intentions to improve the mobility of elderly people is expected to increase $1,2,3,5,6$. THA is intended not only to relieve pain but also to restore hip biomechanics. The restoration of hip biomechanics leads to a minimal number of functional deficits, secures the longevity of the implant, and improves quality of life, mobility, joint stability and locomotion ${ }^{2}, 7$. THA is very effective in alleviating pain, but functional deficits persist up to a year following surgery ${ }^{3}$. Addressing these functional deficits is increasingly important, and postoperative center- and home-based programs have proven beneficial ${ }^{5}$. As technology and surgical techniques have improved, patient expectations from THA have also increased, including an early return to normal physical activities and a recovery of functional independence ${ }^{8}$. Early targeted rehabilitation has been shown to reduce hospital length of stay (LOS) without increasing the complication rates after $\mathrm{THA}^{7},{ }^{910},{ }^{11}$. Utilization of the direct anterior approach, which is performed in the internervous and intermuscular plane, enables a fast recovery with little pain after surgery and does not require postoperative precautions ${ }^{12}$. With the implementation of optimal multimodal perioperative care to enhance recovery, the average hospital LOS has additionally reduced ${ }^{13}$, $14,15,16,17$. A reduction in the hospital LOS has increased the need for an efficient exercise program beyond the initial standard rehabilitation program completed during hospitalization ${ }^{6}$. A disadvantage of these programs is the need for patients to exercise under the supervision of professional staff at a hospital or rehabilitation center. These programs are expensive due to the high costs associated with staff supervision, treatment and transportation between locations and are unfriendly because of the need for transportation ${ }^{6}, 7$. Although there are many studies that have tested different rehabilitation protocols against the "standard" practice, no explicit definition of standard practice exists. Standard physiotherapy rehabilitation programs may comprise hip joint mobilization, strengthening of adjacent muscles without external loading or with low-resistance weight and gait training ${ }^{1}, 2,3,{ }^{18}$. However, it is well known that regardless of a patient's adherence to standard physiotherapy rehabilitation programs, significant additional muscular atrophy and weakness occurs in his or her affected limb, which is often in deficit compared to the healthy limb in patients with unilateral OA prior to surgery $1,37,11$. Suetta et al found $13 \%$ and $9 \%$ reductions in the cross-sectional area of the quadriceps on the operated side at 5 and 13 weeks after THA, respectively, following the completion of a standard program ${ }^{11}$. Deficits in strength have serious adverse consequences for THA patients with respect to physical function, the maintenance of independence and the requirement for revision surgery. Leg strength deficits have been associated with poor gait symmetry, slow walking speeds, impaired stair-climbing and chair-rising abilities, limited access to public transportation, and an exacerbated risk of falling and loosening of the prosthesis ${ }^{1}, 2,47,1119$, 20. In contrast, progressive resistance training (PRT) is an effective method for inducing muscle hypertrophy and increasing muscle strength and functional performance in healthy and clinical populations, including elderly individuals ${ }^{21}$. PRT in rehabilitation following THA has been shown to 
significantly enhance muscle strength and function, and PRT has been shown to be the main factor in achieving significant functional improvements in rehabilitation programs used after home- or centerbased programs after THA $2,5,1122$. First, studies with strength training programs after THA were conducted with patients who underwent surgery after femoral neck fractures ${ }^{18}, 23$. The key points of these studies were that PRT is safe and effective in geriatric rehabilitation after hip surgery; the patients' strength, functional performance and emotional state improve by physical training; and long-term continuation of the programs may prevent detraining effects ${ }^{23}$. The fundamental principle of PRT is to progressively overload the exercised muscle as it becomes stronger ${ }^{22},{ }^{24}$. Evidence-based recommendations for resistance exercise to improve strength and power are as follows: an exercise frequency of 2-3 times per week, an exercise intensity of 60-70\% 1-repetition maximum (1-RM) for novice to intermediate exercisers, 8-12 repetitions, 2-4 sets, a rest interval of 2-3 minutes between sets with $\geq 48$ hours between sessions, and a gradual progression ${ }^{21},{ }^{22}$. Studies in patients who underwent elective THA because of unilateral OA had small sample sizes, but they indicated that there are benefits of strength training protocols, and no major adverse effects were noticed ${ }^{2}, 8,11,19,22,25$. Different strength training protocols were used at different times in the postoperative phase, either in group or individual practices, with major differences being in center- and home-based programs with or without supervision. Some studies comparing supervised home- and center-based rehabilitation programs for THA patients have found greater improvements in function and quality of life for home-based patients than for center-based patients $[7,26,277$. Studies of unsupervised home-based exercise programs beyond the immediate postoperative rehabilitation period have also reported increases in hip muscle strength, walking speed and function with respect to those in postoperative rehabilitation period ${ }^{7}, 20,28$. Furthermore, a home-based rehabilitation program with supervision in the early postoperative period has been shown to be less expensive and more accessible for THA patients than a center-based program ${ }^{26}$, 28,29

In our hospital, we implemented a rapid recovery protocol for all THA patients in 2011 . We continuously made changes, including improvements in the preoperative preparation of patients and their relatives with a multidisciplinary preoperative education program and the implementation of the direct anterior approach, shortening the patients' hospital LOS from an average of 10 to 3 days and improving patient satisfaction. Inherently, the primary goal is safe mobilization, not functional optimization. To date, only minor changes have been made to the standard physiotherapy protocol, and there is still much room for improvement. Currently, patients receive written and pictorial instructions for standard home-based exercises.

\section{Objectives}

The primary objectives of our study are to evaluate whether an early postoperative home-based strength training protocol is feasible for all elective THA patients, does not cause major adverse effects and can improve patient functional outcomes at 3 months and 1 year following surgery. 
The secondary objectives include assessing patient satisfaction with enhanced targeted physiotherapy and preparing a new standardized rehabilitation protocol with videos that will be effective, safe and accessible to all THA patients.

\section{Methods/design Study design}

The study is a multi-center randomized controlled trial comparing the effect of intensive early postoperative physiotherapy with the current standard of physiotherapy for patients undergoing elective THA for unilateral OA. General Hospital Novo mesto and Valdoltra Orthopedic Hospital are involved in the project, and the standard physiotherapy program for patients after THA is similar in both hospitals. All patients who will receive an anesthesiologist's approval and will already be scheduled for surgery will be assessed for eligibility. They will be invited to participate, will receive oral and written explanations about the trial and will be included in the study after signing a written informed consent form. After admission to the hospital, they will complete the routine preoperative outcome assessment questionnaires (Harris Hip Score (HHS) and 36-Item Short Form Health Survey (SF-36)) and have standard preoperative AP Xrays of both hips taken. Physiotherapists will perform the assessments: physical tests and an isometric muscle strength assessment. In both hospitals, the included patients will be randomized to either the intervention group (IG) or the control group (CG), and the surgeons and patients will be blinded to the randomization process. The procedure will be performed with the direct anterior approach only in the General Hospital Novo mesto by 1 surgeon and in the Valdoltra Orthopedic Hospital by 4 surgeons. Patients will be mobilized on the day of surgery. During the expected LOS of 2-5 days, all patients will receive current standard physiotherapy, oral analgesics and cryotherapy. Patients in the IG will receive extra one-on-one training to learn strength and sensory-motor exercises. If the surgeon applies any limitations for a patient regarding rehabilitation after surgery, the patient will be excluded from the study. At discharge, patients in both groups will receive USB drives with exercise videos, written exercise instructions and a training diary. Patients in the IG will also receive exercise aids for the strength and sensory-motor training. All patients will be followed up with regular phone calls concerning possible complications, completion of the training diary, and the clarity of the exercises. The patients will visit the hospital as usual after 1, 3 and 12 months following surgery. At each follow-up, the physiotherapists will perform assessments, and patients will fill out questionnaires. The patients will have a standard postoperative X-ray of the operated hip immediately after the surgery and AP X-rays of both hips after 1 year, so we will be able to compare measurements of the hip offsets of the operated and healthy hips and observe any changes regarding component position or osteolysis. At every follow-up, the surgeon and physiotherapist will ask the patient about any adverse effects or reasons for training cessation. The trial flowchart is displayed in Fig. 1.

\section{Study population}

Inclusion criteria

1. Patients undergoing elective primary unilateral THA for OA. 
2. Patients older than 60 years at the time of surgery.

3. Patients with the ability to watch exercise videos on a USB drive.

4. Patients with an American Society of Anesthesiologists (ASA) Classification 1-3.

5. Patients able to consent to and willing to comply with the study protocol.

\section{Exclusion criteria}

1. Patients undergoing primary THA for a diagnosis other than OA (aseptic femoral head necrosis, dysplastic hip, or others) or revision THA.

2. Patients with previous hip interventions (osteosynthesis, osteotomy...)

3. Patients discharged to rehabilitation units or nursing homes.

4. Patients unable to consent and comply with the study protocol (diagnosed with dementia, mental disorders, poor preoperative physical status, neurological disorders, amputations, trouble walking with walkers or wheelchairs, or a painful hip or knee prosthesis in other joints).

\section{Randomization procedures}

Randomization will be made using a bespoke web-based randomization protocol. Patients will be randomized on a 1:1 ratio to intervention group $A$ (with strength and sensory-motor training included) or control group B (with standard of care physiotherapy), regarding inclusion order. Physiotherapists and coordinators of the trial will be aware of the randomization procedure. Patients and orthopedic surgeons will be blinded, but they will be unblinded, if any safety issue or adverse effect arises.

Sample size calculation

A power calculation was performed based on a clinically significant difference in gait speed of $0.10 \mathrm{~m} / \mathrm{s}$ on the 4- or 10-Meter Walk (10MW) test using data from Perera et al ${ }^{30}$. Based on a substantial meaningful change of $0.10 \mathrm{~m} / \mathrm{s}$ between the interventional and control groups with a significance level of $5 \%$ and a power of $80 \%$, a needed sample size of 124 patients in each group was estimated. All patients from surgeons, participating in the study, will be assessed for eligibility.

\section{Study intervention}

Standard rehabilitation

All patients will receive standard of care physiotherapy during hospitalization. Physiotherapy will commence on the day of surgery with mobilization using a walking aid (usually two crutches, rarely a walker), deep vein thrombosis prevention exercises, lower limb range of motion exercises and an isometric strengthening program. Patients will be encouraged to perform exercises twice daily, with approximately 10 repetition s overy exercise, and walk as much as possible. Exercises will be performed in a supine position, on the healthy side, on the abdomen, sitting and standing (e.g., buttock squeezes, leg sliding motions, straight leg raises, bridges, postural exercises). Patients will be discharged based on a combination of compliance with the exercises, stability with a walking aid, independence with activities 
of daily living and dry wounds. Patients in the CG will receive written instructions, a USB drive with videos of standard exercises and a training dairy at discharge.

Prescribed home-based strength and sensory-motor training

Before the patients are discharged, physiotherapists will teach patients in the IG special strength and sensory-motor exercises with exercise aids. The patients will also receive USB drives with special exercise videos, written exercise instructions and a training diary to check performed exercises. The new training protocol includes exercises for improving hip stability and reducing local stresses on the hip prosthesis. In the training, we considered safety regarding hip and lumbar spine load, falls and prosthesis luxation. Training will consist of: hip muscle strengthening exercises (focusing on the abductors); hip and pelvic stabilization exercises; ankle and knee muscle strengthening exercises (for improved dissipation of impact forces and femoral inner rotation control); and trunk muscle strengthening exercises (for stabilization of the pelvis and lumbar spine and a reduction in local loads). We ensured the exercises are simple and easy to understand, the videos that are to be watched at home are easy to follow, and the training program does not require expensive equipment. In the first two postoperative weeks, the focus is on learning the proper exercise technique and developing a sense of loading. Patients will watch special instructional short videos with exercise descriptions. Each exercise will be demonstrated with easy and challenging options as well as oral and written instructions. We will use a revised Borg category-ratio scale ( 0 to 10 scale) for perceived exertion to monitor and guide exercise intensity ${ }^{31}$. In sets of fluent concentric repetitions of movements (10-12 repetitions per set), the rating of perceived exertion will be recorded for the last repetition. When holding an isometric position, the rating will be recorded for the end of interval (with a lengthening of the interval to 30 seconds, until we can achieve desired perceived exertion at the end of the interval). In sets of isometric contractions, every repetition will be made with maximal voluntary effort exerted for 6 seconds $(2$ seconds for force increments and 4 seconds for maintenance). The implementation of fluent concentric movements is intended to increase muscle mass and improve physical endurance, while maximal isometric contractions are used for improving the level of muscular activation ${ }^{32}$. Exercises with isometric trunk positions will be prepared with recommendations for isometric trunk stabilization training ${ }^{33}$. These concepts collectively allow for individual load adaption for every exercise regarding individual patient abilities and load progression in accordance with his or her progress in muscle strengthening training ${ }^{32}$. Each session consists of a warm-up with 2 options (5-7 minutes of toe walking on the same spot or step-ups on a $10 \mathrm{~cm}$ tall step), strength training for both legs (30-40 minutes; 11 exercises; $1-4$ fluent concentric repetitions of the single-leg squat and lying side hip raise; 5-8 maximal isometric contractions of the sitting ball squeeze, seated elastic band hip abduction, and diagonal ball push into the opposite thigh; 9-11 holds in the isometric positions, side plank and bridge) and stretching (standing hip flexor stretch, sitting forward bend stretch, standing lateral trunk stretch). Strength training will be performed twice weekly, with a minimum of 2 days of rest. Until the 5th postoperative week, perceived exertion will be increased on the Borg scale from 5 to 8 , and then it will be increased to 9 until the assessment at 3 months. In the first 5 postoperative weeks, 2 sets of each exercise will be recommended, and after these 5 weeks, 3 sets will be 
recommended. Sensory-motor training will be added to strength training to increase muscular activation and rate of force development and will be practiced on the days without strength training ${ }^{34}$. Exercise for ankle stability will be performed with a one-dimensional wooden balance board in the frontal and sagittal plane ${ }^{35}$. At the beginning, patients will perform 6 sets for each leg and 12 sets after the 7th postoperative week. The intensity of the exercise will be increased according to patient abilities with changes in the stability of the balance board. For every exercise, severe pain will be a reason for training cessation and contact with the physiotherapist.

\section{Outcome measures}

This paper is written according to the Standard Protocol Items: Recommendations for Interventional Trials (SPIRIT) 2013 Statement for the reporting of clinical trial protocols (Table 1, Additional file 1) ${ }^{36}, 37$. The primary outcomes are those of the physical tests, which comprise isometric muscle strength, the $10 \mathrm{MW}$ test and the 8-foot Up and Go (8UG) test. Secondary outcomes are the patient reported outcome measures: HHS and SF-36. 
Table 1

SPIRIT-Figure ${ }^{36}, 37$ : Schedule and outcome measurements preoperatively and at 1, 3 and 12 months postoperatively

\begin{tabular}{|llllll|}
\hline Timepoint & Preop & Discharge & 1 month & 3 months & 1 year \\
\hline ENROLLMENT: & & & & \\
\hline Eligibility screen & $\mathrm{X}$ & & & \\
\hline Informed consent & $\mathrm{X}$ & & & \\
\hline Group allocation & $\mathrm{X}$ & & & \\
\hline INTERVENTIONS: & & & & & \\
\hline Intervention group (IG) & & $\mathrm{X}-\mathrm{X}$ & \\
\hline Control group (CG) & & $\mathrm{X}-\mathrm{X}$ & \\
\hline ASSESSMENTS: & & & & & \\
\hline Patient demographics & $\mathrm{X}$ & & $\mathrm{X}$ & $\mathrm{X}$ & $\mathrm{X}$ \\
\hline Isometric muscle strength & $\mathrm{X}$ & & $\mathrm{X}$ & $\mathrm{X}$ & $\mathrm{X}$ \\
\hline 8UG & $\mathrm{X}$ & & $\mathrm{X}$ & $\mathrm{X}$ & $\mathrm{X}$ \\
\hline 10MW & $\mathrm{X}$ & & $\mathrm{X}$ & $\mathrm{X}$ & $\mathrm{X}$ \\
\hline HHS & $\mathrm{X}$ & & $\mathrm{X}$ & $\mathrm{X}$ & $\mathrm{X}$ \\
\hline SF-36 & $\mathrm{X}$ & $\mathrm{X}$ & $\mathrm{X}$ & & \\
\hline X rays & & & & $\mathrm{X}$ \\
\hline Complications & & & & \\
\hline
\end{tabular}

Trial baseline is on the day of hospital admission, one day before surgery. Assessment timeframes are calculated from the date of surgery.

Maximal voluntary isometric contractions

We use a specially prepared measuring device with a dynamometer attached to stiff band metal chains, prepared similarly to those in Roussel et al and Essendrop et al ${ }^{38}, 39$. The ICC for a similar device, used by one of the senior authors for lumbar muscle strength assessment, is $>0.94$. For the assessment of maximal isometric strength, a maximal voluntary isometric contraction for 6 seconds is used. The same procedure of obtaining a maximal voluntary contraction is used for every strength measure. Movement is assessed in 3 planes for the trunk (extension, left and right abduction) and operated hip (flexion, extension and abduction), and movement is assessed in 2 planes for the contralateral hip (extension, abduction). All strength tests are performed in a neutral standing position, and the patient rotates on the measuring device so that the exerted force is always in the direction opposite of the dynamometer. Before 
the measurements, the height of pelvic support is set to the reference point of the iliac crest of each patient. Patients are verbally encouraged to generate maximal effort. The mean duration of the complete test is 20 minutes. We wrap the band around the trunk under the armpits for trunk tests and around the ankle for leg tests. We measure the height of pelvic support, maximal contraction force and distance between the upper border of the support board and the middle point of the stiff band. All measurements are taken twice.

Physical performance tests

We will make videos of patients performing both physical tests, and later, one independent researcher will review them. All tests will be performed twice. If possible, the patient will walk without walking aids; if a patient is unable to perform a test without help, this instance will be allowed and noted.

\section{8-FOOT UP AND GO TEST}

For the Timed Up and Go (TUG) test, the time that a person takes to rise from a chair, walk 3 meters, turn around, walk back to the chair, and sit down is measured ${ }^{40}$. This test is used to assess mobility, balance, walking ability and fall risk in elderly populations. The original version of the TUG test has been critiqued because of the potential for confusion with its instructions and the line on the floor. To address this limitation, Rikli and Jones modified the TUG test to create the $8 U G$ test by reducing the distance to 8 feet and replacing the line on the floor with a cone to signal the turn-around point 41,4243 . The 8UG test has better sensitivity than the TUG test when traditional cut-off times are applied. The 8UG test with a cut off time of 8.5 seconds should be used as a threshold for identifying persons with an increased risk of falling 42,43 . The 8 UG test is a timed test that requires one to stand up from a chair with armrests, walk to a cone 8 feet away, turn around, return to the chair and sit down ${ }^{42}$.

\section{TIMED 10-METER WALK TEST}

The speed at which individuals walk is relevant to their functional abilities in the community ${ }^{30,44}$. Gait speed is also an important predictor of outcomes, such as hospital LOS and discharge disposition of patients admitted for acute rehabilitation after a stroke, mortality of older adults, incident ischemic stroke among postmenopausal women and incident dementia among older adults ${ }^{44}$. For this test, the individual walks 10 meters without assistance, and the time the individual takes to walk the intermediate 6 meters is measured to allow for acceleration and deceleration periods ${ }^{45}$. We measure and mark a 10-meter walkaway and add a mark at 2 and 8 meters. The test is first performed at preferred walking speed and performed a second time at the fastest speed possible ${ }^{45}$.

Questionnaires

HARRIS HIP SCORE 
The HHS is a widely used disease-specific measure of hip disabilities after THA. The physiotherapist administers the test in the form of a structured interview with the patients. The domains include pain, functions of daily living and gait. The rating scale is from 0 (worse) to 100 points (best) ${ }^{46}$. The HHS is considered to have good validity and reliability ${ }^{47}$.

\section{6-ITEM SHORT FORM HEALTH SURVEY}

The SF-36 Health Survey was first made available in standard form in 1990. The eight health domains represented in the profile were selected from the 40 domains that were included in the Medical Outcome Study (MOS) by Stewart and Ware ${ }^{48}$. The RAND 36-Item Health Survey (Version 1.0) is a set of generic, coherent, and easily administered quality-of-life measures. It taps eight health concepts: physical functioning, bodily pain, role limitations due to physical health problems, role limitations due to personal or emotional problems, emotional well-being, social functioning, energy/fatigue, and general health perceptions. It also includes a single item that provides an indication of perceived change in health ${ }^{49}$. These 36 items, presented in RAND 36-Item Health Survey 1.0, are identical to the MOS SF-36 described in Ware and Sherbourne ${ }^{50}$. Scoring of the RAND 36 -Item Health Survey 1.0 is clearly described in scoring instructions ${ }^{49}$. The survey is a practical, reliable and valid measure of physical and mental health. In our study, we will use the Slovenian translated and validated version by Marn-Vukadinović et al ${ }^{51}$.

Training compliance

All participants in both groups will keep a training dairy concerning all training activities; they will make marks by every exercise they perform on each day, write notes if they will perform any other sport activities (walking, stationary bicycling and others), note any adverse effects, and note reasons for skipped training exercises if applicable. They will also record their pain level by using the Visual Analog Scale (VAS) ${ }^{52}$ during the day, before and after training, and during the night.

\section{Statistical analysis}

Software program SPSS 21 (IBM SPSS Statistics for Windows, Version 21.0. Armonk, NY: IBM Corp.) will be used. We will test variables for normality of distribution by the Kolmogorov-Smirnov test (K-S test), which is a nonparametric test of the equality of continuous one-dimensional probability distributions. When using the K-S test for testing normality of distributions, samples are standardized and compared with standard normal distributions. With normally distributed values, we will use parametric tests: analysis of variance (ANOVA) for testing differences in the effects of two different exercise protocols and analyzing between-group differences in the change scores from baseline to the different time points; the paired samples t-test for in-group differences in the change scores from the baseline to the end point; the independent samples t-test for between-group comparisons at the same time point; the bivariate Pearson correlation for the evaluation of relationships among changes in different values and between changes in values and baseline values in both groups. To test the reliability of maximal voluntary isometric contraction measurements and the 8UG test, we will use the intraclass correlation coefficient (ICC) with the two-way mixed-effects model with absolute agreement. Data will be presented as the arithmetic mean 
and standard deviation. In the case of a distorted distribution, data will be presented as the median and interquartile range, and nonparametric tests will be used for comparisons of dependent and independent samples. With the chi-square test, we will compare differences between groups regarding patient satisfaction questionnaires and adverse effects. We will consider a $P$ value less than 0.05 as significant for all measures and use a $95 \%$ confidence interval (Cl). A significance level (alpha) of 0.05 will be tested with a two-tailed test.

\section{Discussion}

We have had good experiences with the standard exercise protocol, so we anticipate that the majority of the patients from both groups will have better performance at 3 months and 1 year after surgery compared to at baseline. All patients should also have higher scores on patient satisfaction questionnaires at 3 months and 1 year after surgery than at baseline. We expect patients in the intervention group to achieve better results in physical tests and maximal voluntary isometric contraction measurements compared to patients in the control group. With respect to other studies, we predict the largest differences at the 3rd postoperative month. We would like to provide evidence that home-based strength and sensory-motor training is safe and easy to understand for the majority of patients and is applicable to all THA patients.

\section{Future directions}

The aim of our project is to design a safe, affordable home-based strength and sensory-motor training program for all THA patients that can integrate well with the existing rapid recovery protocol in our hospitals.

\section{Trial Status}

Patient recruitment for our study commenced in January 2018 in the General Hospital Novo mesto and in April 2018 in the Valdoltra Orthopedic Hospital and is on-going at the time of the manuscript submission. The expected time of recruitment completion is June 2021.

\section{Abbreviations}

THA - Total hip arthroplasty

PRT - Progressive resistance training

IG - Intervention group

CG - Control group

HHS - Harris Hip Score 
SF-36 - 36-Item Short Form Health Survey

OA - Osteoarthritis

LOS - Length of stay

1-RM - 1-repetition maximum

10MW - 10-Meter Walk

8UG - 8-foot Up and Go

TUG - Timed Up and Go

ASA - American Society of Anesthesiologists

VAS - Visual Analog Scale

ICC - intraclass correlation coefficient

\section{Declarations}

\section{Ethics approval and consent to participate}

The study received ethical approval from the National Medical Ethics Committee of Ministry of Health, Republic of Slovenia (approval number: 0120-388/2017-4 KME 47/07/17). All participants will sign approved informed consent forms.

\section{Consent for publication}

The authors will obtain consent from the participants to publish individual patient data without personal details.

\section{Availability of data and materials}

The datasets used and analyzed during the current study are available from the corresponding author upon reasonable request.

\section{Competing interests}

The authors declare that they have no competing interests.

\section{Funding}

There is no funding for the research.

\section{Authors' contributions}


The protocol was developed by PKM, VS and RT. All authors read and approved the final manuscript.

\section{Acknowledgements}

The authors thank the physiotherapists for performing all physical tests and supporting the implementation of the study.

\section{References}

1. Lemmey A, Okoro T. The efficacy of exercise rehabilitation in restoring physical function following total hip replacement for osteoarthritis: a review. OA Musculoskeletal Med. 2013;1:13.

2. Husby VS, Helgerud J, Bjørgen S, Husby OS, Benum P, Hoff J. Early maximal strength training is an efficient treatment for patients operated with total hip arthroplasty. Arch Phys Med Rehabil. 2009;90(10):1658-67.

3. Okoro T, Ramavath A, Howarth J, et al. What does standard rehabilitation practice after total hip replacement in the UK entail? Results of a mixed methods study. BMC Musculoskelet Disord. 2013;14(1):91.

4. Di Monaco M, Castiglioni C. Which type of exercise therapy is effective after hip arthroplasty? A systematic review of randomized controlled trials. Eur J Phys Rehabil Med. 2013;49(6):893-907. quiz 921-3.

5. Okoro T, Morrison V, Maddison P, Lemmey A, Andrew J. An assessment of the impact of behavioural cognitions on function in patients partaking in a trial of early home-based progressive resistance training after total hip replacement surgery. Disabil Rehabil. 2013;35(23):2000-7.

6. Monaghan B, Grant T, Hing W, Cusack T. Functional exercise after total hip replacement (FEATHER) a randomised control trial. BMC Musculoskelet Disord. 2012;13(1):237.

7. Galea MP, Levinger P, Lythgo N, et al. A targeted home-and center-based exercise program for people after total hip replacement: a randomized clinical trial. Arch Phys Med Rehabil. 2008;89(8):1442-7.

8. Wang AW, Gilbey HJ, Ackland TR. Perioperative exercise programs improve early return of ambulatory function after total hip arthroplasty: a randomized, controlled trial. American journal of physical medicine $\backslash \&$ rehabilitation. 2002;81(11):801-6.

9. Larsen K, Hvass KE, Hansen TB, Thomsen PB, Søballe K. Effectiveness of accelerated perioperative care and rehabilitation intervention compared to current intervention after hip and knee arthroplasty. A before-after trial of 247 patients with a 3-month follow-up. BMC Musculoskeletal disorders. 2008;9(1):59.

10. Larsen K, Hansen TB, Søballe K. Hip arthroplasty patients benefit from accelerated perioperative care and rehabilitation: a quasi-experimental study of 98 patients. Acta Orthop. 2008;79(5):624-30.

11. Suetta C, Magnusson SP, Rosted A, et al. Resistance training in the early postoperative phase reduces hospitalization and leads to muscle hypertrophy in elderly hip surgery patients-a controlled, randomized study. J Am Geriatr Soc. 2004;52(12):2016-22. 
12. Moskal JT, Capps SG, Scanelli JA. Anterior muscle sparing approach for total hip arthroplasty. World journal of orthopedics. 2013;4(1):12.

13. Husted H, Lunn TH, Troelsen A, Gaarn-Larsen L, Kristensen BB, Kehlet H. Why still in hospital after fast-track hip and knee arthroplasty? Acta Orthop. 2011;82(6):679-84. doi:10.3109/17453674.2011.636682.

14. Husted H, Gromov K, Malchau H, Freiberg A, Gebuhr P, Troelsen A. Traditions and myths in hip and knee arthroplasty: A narrative review. Acta Orthop. 2014;85(6):548-55.

15. Kehlet $\mathrm{H}$, Wilmore DW. Evidence-based surgical care and the evolution of fast-track surgery. Ann Surg. 2008;248(2):189-98. doi:10.1097/SLA.0b013e31817f2c1a.

16. Kehlet $\mathrm{H}$, Wilmore DW. Multimodal strategies to improve surgical outcome. Am J Surg. 2002;183(6):630-41.

17. Den Hartog YM, Mathijssen NMC, Vehmeijer SBW. Reduced length of hospital stay after the introduction of a rapid recovery protocol for primary THA procedures. Acta Orthop. 2013;84(5):4447. doi:10.3109/17453674.2013.838657.

18. Hesse S, Werner $C$, Seibel $H$, et al. Treadmill training with partial body-weight support after total hip arthroplasty: a randomized controlled trial. Arch Phys Med Rehabil. 2003;84(12):1767-73.

19. Mikkelsen LR, Mikkelsen SS, Christensen FB. Early. Intensified Home-based Exercise after Total Hip Replacement-A Pilot Study. Physiotherapy research international. 2012;17(4):214-26.

20. Jan M-H, Hung J-Y, Lin JC-H, Wang S-F, Liu T-K, Tang P-F. Effects of a home program on strength, walking speed, and function after total hip replacement. Arch Phys Med Rehabil. 2004;85(12):194351.

21. Garber CE, Blissmer B, Deschenes MR, et al. American College of Sports Medicine position stand. Quantity and quality of exercise for developing and maintaining cardiorespiratory, musculoskeletal, and neuromotor fitness in apparently healthy adults: guidance for prescribing exercise. Med Sci sports Exerc. 2011;43(7):1334-59.

22. Mikkelsen LR, Petersen AK, Mechlenburg I, Mikkelsen S, Søballe K, Bandholm T. Description of load progression and pain response during progressive resistance training early after total hip arthroplasty: secondary analyses from a randomized controlled trial. Clinical rehabilitation. 2017;31(1):11-22.

23. Hauer K, Specht N, Schuler M, Bärtsch P, Oster P. Intensive physical training in geriatric patients after severe falls and hip surgery. Age Ageing. 2002;31(1):49-57.

24. DeLorme T, Watkins A. Technics of progressive resistance exercise. Arch Phys Med Rehabil. 1948;29(5):263.

25. Skoffer B, Dalgas U, Mechlenburg I. Progressive resistance training before and after total hip and knee arthroplasty: a systematic review. Clinical rehabilitation. 2015;29(1):14-29.

26. Siggeirsdottir K, Olafsson Ö, Jonsson H Jr, Iwarsson S, Gudnason V, Jonsson BY. Short hospital stay augmented with education and home-based rehabilitation improves function and quality of life after 
hip replacement: randomized study of 50 patients with 6 months of follow-up. Acta Orthop. 2005;76(4):555-62.

27. Mahomed NN, Davis AM, Hawker G, et al. Inpatient compared with home-based rehabilitation following primary unilateral total hip or knee replacement: a randomized controlled trial. JBJS. 2008;90(8):1673-80.

28. Chang C-F, Lin K-C, Chen W-M, Jane S-W, Yeh S-H, Wang T-J. Effects of a Home-Based Resistance Training Program on Recovery From Total Hip Replacement Surgery: Feasibility and Pilot Testing. journal of nursing research. 2017;25(1):21-30.

29. Okoro T, Whitaker R, Gardner A, Maddison P, Andrew JG, Lemmey A. Does an early home-based progressive resistance training program improve function following total hip replacement? Results of a randomized controlled study. BMC Musculoskelet Disord. 2016;17(1):173.

30. Perera S, Mody SH, Woodman RC, Studenski SA. Meaningful change and responsiveness in common physical performance measures in older adults. J Am Geriatr Soc. 2006;54(5):743-9.

31. Borg GA. Psychophysical bases of perceived exertion. Med sci sports exerc. 1982;14(5):377-81.

32. Schmidtbleicher D. Klassifizierung der Trainingsmethoden im Krafttraining. Lehre der Leichtathletik. 1985;24(1):2.

33. McGill SM. Low back disorders: evidence-based prevention and rehabilitation. Human Kinetics; 2015.

34. Taube W, Gruber M, Gollhofer A. Spinal and supraspinal adaptations associated with balance training and their functional relevance. Acta Physiol (Oxf). 2008;193(2):101-16. doi:10.1111/j.17481716.2008.01850.x.

35. Gruber M, Gollhofer A. Impact of sensorimotor training on the rate of force development and neural activation. European journal of applied physiology. 2004;92(1-2):98-105.

36. Chan A-W, Tetzlaff JM, Altman DG, et al. SPIRIT 2013 statement: defining standard protocol items for clinical trials. Ann Intern Med. 2013;158(3):200-7. doi:10.7326/0003-4819-158-3-201302050-00583.

37. Chan A-W, Tetzlaff JM, Gøtzsche PC, et al. SPIRIT 2013 explanation and elaboration: guidance for protocols of clinical trials. Bmj. 2013;346.

38. Roussel NA, Truijen S, De Kerf I, Lambeets D, Nijs J, Stassijns G. Reliability of the assessment of lumbar range of motion and maximal isometric strength in patients with chronic low back pain. Arch Phys Med Rehabil. 2008;89(4):788-91.

39. Essendrop M, Schibye B, Hansen K. Reliability of isometric muscle strength tests for the trunk, hands and shoulders. Int J Ind Ergon. 2001;28(6):379-87.

40. Timed Up and Go Instructions. Available at: https://www.sralab.org/rehabilitation-measures/timedand-go. Accessed 6, 2020.

41. Rikli RE, Jones CJ. Development and validation of criterion-referenced clinically relevant fitness standards for maintaining physical independence in later years. Gerontologist. 2013;53(2):255-67.

42. MSPT JCR, others. Validity of the 8-Foot Up and Go, Timed Up and Go, and Activities-Specific Balance Confidence scale in older adults with and without cognitive impairment. J Rehabil Res Dev. 
2016;53(4):511.

43. Rose DJ, Jones CJ, Lucchese N. Predicting the probability of falls in community-residing older adults using the 8-foot up-and-go: a new measure of functional mobility. Journal of Aging Physical Activity. 2002;10(4):466-75.

44. Bohannon RW, Williams Andrews A. Normal walking speed: a descriptive meta-analysis.

Physiotherapy. 2011;97(3):182-9. doi:10.1016/j.physio.2010.12.004.

45. 10 Meter Walk Test. Available at: https://www.sralab.org/rehabilitation-measures/10-meter-walk-test. Accessed 6, 2020.

46. Harris WH. Traumatic arthritis of the hip after dislocation and acetabular fractures: treatment by mold arthroplasty. An end-result study using a new method of result evaluation. J Bone Joint Surg Am. 1969;51(4):737-55.

47. Só§derman $\mathrm{P}$, Malchau $\mathrm{H}$. Is the Harris hip score system useful to study the outcome of total hip replacement? Clin Orthop Relat Res. 2001;384:189-97.

48. Ware JE. SF-36 health survey: manual and interpretation guide. Health Institute. 1993.

49. 36-Item Short Form Survey (SF-36). Available at: https://www.rand.org/healthcare/surveys_tools/mos/36-item-short-form.html. Accessed 6, 2020.

50. Ware JE, Sherbourne CD. The MOS 36-item short-form health survey (SF-36). I. Conceptual framework and item selection. Med Care. 1992;30(6):473-83.

51. Marn-Vukadinovic D, Jamnik H. Validation of the short form-36 health survey supported with isokinetic strength testing after sport knee injury. Journal of sport rehabilitation. 2011;20(3):261-76.

52. Bijur PE, Silver W, Gallagher EJ. Reliability of the visual analog scale for measurement of acute pain. Acad Emerg Med. 2001;8(12):1153-7.

\section{Figures}




\section{Pre-op Assessment}

Assessed for eligibilitv, Inclusion/Exclusion criteria

Muscle strength, 8UG, 10MW, HHS, SF-36, X-rav, Randomisation

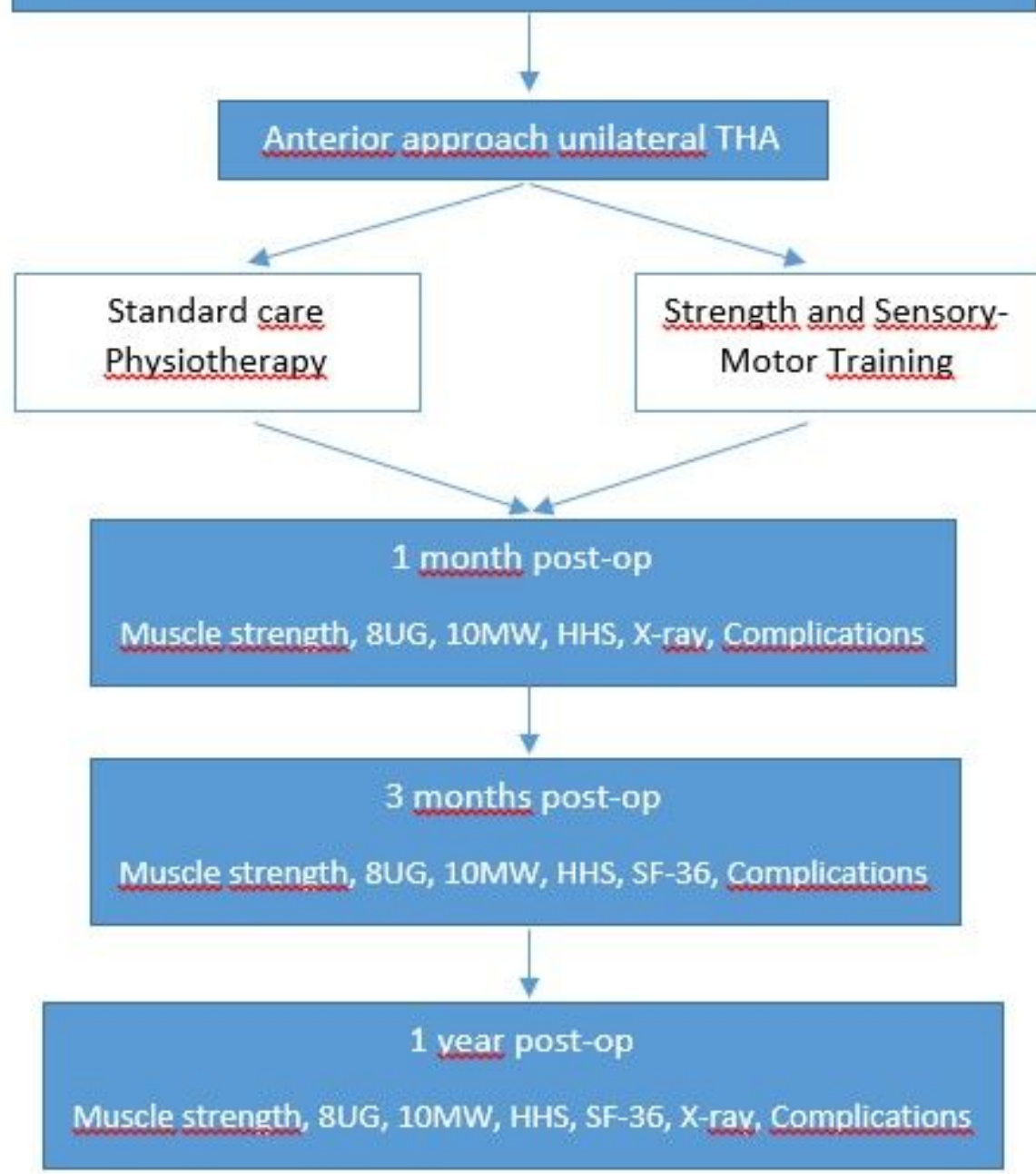

\section{Figure 1}

Trial flowchart

\section{Supplementary Files}

This is a list of supplementary files associated with this preprint. Click to download.

- SPIRITchecklistPKM.docx 